To appear in the International Journal of Computational Fluid Dynamics Vol. 00, No. 00, 00 Month 20XX, 1-8

\title{
Investigating cross-wind stability of high speed trains with large-scale parallel CFD
}

\author{
M.M. Fragner ${ }^{\mathrm{a} *}$ and R. Deiterding ${ }^{\mathrm{b}}$ \\ ${ }^{a}$ BTU Cottbus - Senftenberg \\ Siemens-Halske-Ring 14, 03046 Cottbus, Germany \\ ${ }^{b}$ University of Southampton \\ Highfield Campus, Southampton SO17 1BJ, United Kingdom
}

( $v 1.1$ released June 2016)

\begin{abstract}
The side-wind loading on a simplified train model at scale 1:25 is investigated by parallel LES with incompressible solvers from the OpenFOAM package and a novel dynamically adaptive, parallel LEStype lattice Boltzmann method implemented in our own AMROC framework. It is found that the new LBM code provides more accurate time-averaged force predictions, while compute times are reduced.
\end{abstract}

Keywords: Train aerodynamics; Large eddy simulation (LES); Lattice Boltzmann method (LBM); Adaptive mesh refinement; Pressure-correction method; Delayed detached eddy simulation (DDES)

\section{INTRODUCTION}

Modern high speed passenger trains are at risk of derailment when operating in strong cross winds. Most experimental studies utilizing wind tunnel experiments have focused on measuring integral forces, such as drag and lift coefficients and the overturning moment, e.g. (Baker et al. 2004). Computational fluid dynamics (CFD) on the other hand potentially allows for a much more detailed understanding of the flow behavior. For larger yaw angles, that is for inflows at an angle larger $20^{\circ}$, the flow exhibits unsteady vortex shedding from the roof as well as the underside of the train and it is vital to employ a turbulence modeling approach that is capable of resolving at least partially the turbulent dynamics of the flow.

Using a simplified model without inter-car gaps and wheel bogies, Hemida and Krajnovic (2010) were the first to use large eddy simulations to model the time-dependent cross-wind flow around train-shaped bodies. Focusing on the influence of nose shape and yaw angle on flow behavior, they found that the nose of the train renders the flow three-dimensional at least for a distance of up to 3.5 trains heights from the tip of the nose in the direction of the length of the train.

In the current investigation, the more realistic Next Generation Train 2 (NGT2) model (Winter 2013) of the DLR at a scale of 1:25 is investigated by LES. This wind tunnel model consists of the front car and a streamlined body intended to replicate the aerodynamic influence of following cars. We concentrate exclusively on a wind impact angle of $\beta=30^{\circ}$ and Reynolds number $\operatorname{Re}=250,000$ (based on the nominal height of the train) for which experimentally obtained temporally averaged force measurements on the front car are available (Fragner et al. 2014).

Because of the excessive computational demand typically associated with LES, we have used in here - beside conventional incompressible LES/DDES Navier-Stokes (NS) solvers from the Open-

*Corresponding author. Email: moritz.fragner@b-tu.de 
FOAM (OF) package - a novel in-house adaptive lattice Boltzmann method with LES-type modeling implemented in the block-based AMROC framework (Deiterding 2011). The LBM is an explicit time-exact scheme for weakly compressible flows that is based on a Cartesian lattice grid. Complex boundaries are treated efficiently with an embedded boundary approach. In our implementation, local mesh adaptation is used to increase resolution around geometric objects such that boundary layer behavior is correctly represented.

The paper is organized as follows: In Section 2, a brief overview of the employed solvers is given. Section 3 describes the benchmark setup and the utilized meshes. The results are discussed in Section 4 in terms of flow topology and calculated force coefficients. The conclusions are given in Section 5. It is generally found that while the LBM-LES results appear to be more instationary, the time-averaged LBM force predictions are closer to experimental measurements and the method is computationally significantly less expensive than pressure-correction based solvers.

\section{COMPUTATIONAL METHODS}

\subsection{OpenFOAM}

We employ LES and DDES for turbulence modeling together with the implicit transient solver pimpleFoam from the OpenFOAM package. In LES, the large eddies are resolved directly and the influence of small-scale eddies on the large scales is modeled. This is achieved by applying a spatial filter to the incompressible NS equations, yielding

$$
\partial_{t} \overline{\mathbf{u}}+\overline{\mathbf{u}} \cdot \nabla \overline{\mathbf{u}}=-\frac{1}{\rho} \nabla \bar{p}+\nu \nabla^{2} \overline{\mathbf{u}}+\nabla \tau^{\mathrm{SGS}}, \quad \nabla \overline{\mathbf{u}}=0 .
$$

Here, $\overline{\mathbf{u}}$ and $\bar{p}$ represent the resolved filtered velocity and pressure, respectively, while $\tau^{\mathrm{SGS}}=$ $\mathbf{u} \mathbf{u}-\overline{\mathbf{u}} \overline{\mathbf{u}}$ are the sub-grid scale (SGS) stresses. Because of its simplicity, we use the Smagorinsky model to describe $\tau^{\mathrm{SGS}}$ for the LES simulations as

$$
\tau_{i j}^{\mathrm{SGS}}=-2 \nu_{t} \bar{S}_{i j} \quad \text { with } \quad \nu_{t}=\left(C_{s} f \Delta x\right)^{2}|\bar{S}|,
$$

where $\overline{S_{i j}}$ is the symmetric part of the strain rate tensor, $C_{S}$ is the Smagorinsky coefficient, $\Delta x$ is the filter width (to be taken as mesh cell size), $|\bar{S}|=\sqrt{2 S_{i j} S_{i j}}$ and $f \approx 1-\exp \left(-\frac{y^{+}}{26}\right)$ is the van Driest damping function, which is used to damp the turbulence length scale next to the wall.

Because of the large resolution requirements in order to properly resolve wall boundary layers with LES at industry typical Reynolds numbers, hybrid models such as detached eddy simulations (DES/DDES) have become a popular alternative to LES (Spalart et al. 2006). DDES methods employ RANS models for $\tau^{\text {SGS }}$ near the wall and transition into LES mode further away by manipulating the model length scale. We use the Spalart-Allmaras (SA) model as the underlying RANS model, while the model length scale $d$ entering the SA model, is being modified according to

$$
d=d_{W}-f_{d} \max \left(0, d_{W}-C_{\mathrm{DES}} \Delta x\right), \quad f_{d}=1-\tanh \left[\left(8 r_{d}\right)^{3}\right], \quad r_{d}=\frac{\nu_{t}+\nu}{\kappa^{2} d_{W}^{2}},
$$

where $d_{W}$ is the closest distance to the wall, $C_{\mathrm{DES}}=0.65$ and $\nu_{t}$ is described by the SA model. Defined in this way, it can be shown that far away from the wall, the model recovers Eq. (2) for $\nu_{t}$. Inside the wall boundary layer the underlying SA model remains unmodified. In OpenFOAM, mainly pressure-correction algorithms are available to approximate the incompressible equations. 


\subsection{Lattice Boltzmann method in AMROC}

The LBM is based on solving for a distribution function $f(\mathbf{x}, t, \mathbf{u})$ the simplified Boltzmann equation $\partial_{t} f+\mathbf{u} \cdot \nabla f=\omega\left(f^{e q}-f\right)$ in discrete phase space. Partial density distribution functions $f_{\alpha}(x, t)$ are associated uniquely to transport in a discrete velocity direction $\mathbf{e}_{\alpha}$. The total density distribution is given as $\rho(\mathbf{x}, t)=\sum_{\alpha} f_{\alpha}(\mathbf{x}, t)$ and the macroscopic moments are $\rho(\mathbf{x}, t) u_{i}(\mathbf{x}, t)=\sum_{\alpha} \mathbf{e}_{\alpha i} f_{\alpha}(\mathbf{x}, t)$. The discrete lattice Boltzmann equation reads

$$
f_{\alpha}\left(\mathbf{x}+e_{\alpha} \Delta t, t+\Delta t\right)=f_{\alpha}(\mathbf{x}, t)+\omega_{L} \Delta t\left(f_{\alpha}^{e q}(\mathbf{x}, t)-f_{\alpha}(\mathbf{x}, t)\right)
$$

where the time step is determined by $\Delta t=\Delta x /\left(\sqrt{3} c_{s}\right)$ based on the speed of sound $c_{s}$, which corresponds to a trivial advection step (aka streaming) of $f_{\alpha}$ in direction of $\mathbf{e}_{\alpha}$ by exactly one point on the uniformly spaced lattice. In this paper, a three-dimensional stencil with 19 distribution functions, i.e., 19 update equations (4) is used (aka D3Q19). For the right hand collision of (4) we adopt the equilibrium function

$$
f_{\alpha}^{e q}(\rho, \mathbf{u})=\rho t_{\alpha}\left[1+\frac{3 \mathbf{e}_{\alpha} \mathbf{u}}{c^{2}}+\frac{9\left(\mathbf{e}_{\alpha} \mathbf{u}\right)^{2}}{2 c^{4}}-\frac{3 \mathbf{u}^{2}}{2 c^{2}}\right] \text { with } t_{0}=\frac{1}{3}, t_{1, \ldots, 6}=\frac{1}{18}, t_{7, \ldots, 18}=\frac{1}{36},
$$

for which via a Chapman-Enskog expansion convergence of the method to a solution of the weakly compressible Navier-Stokes equations

$$
\partial_{t} \rho+\nabla \cdot(\rho \mathbf{u})=0, \quad \partial_{t} \mathbf{u}+\mathbf{u} \cdot \nabla \mathbf{u}=-\frac{1}{\rho} \nabla p+\nu \nabla^{2} \mathbf{u}
$$

can be shown (Hähnel 2004). Kinematic viscosity $\nu$ and discrete collision frequency $\omega_{L}$ are connected by the relation $\omega_{L}=\tau_{L}^{-1}=c_{s}^{2} /\left(\nu+\Delta t c_{s}^{2} / 2\right)$.

To consider high-Re flows, a large eddy approach is adopted, where it is assumed that the LBM above provides filtered resolved distributions $\bar{f}_{\alpha}$ and an eddy viscosity model can applied. The effective viscosity $\nu^{\star}=\nu+\nu_{t}=\frac{1}{3}\left(\tau^{\star} / \Delta t-\frac{1}{2}\right) \Delta x^{2} / \Delta t$ is used with $\tau_{L}^{\star}=\tau_{L}+\tau_{t}=: 1 / \omega_{L}^{\star}$ in (4). Like Hou et al. (1996), we apply the Smagorinsky model to evaluate $\nu_{t}$ as given by (2), but neglect the van Driest damping function. The filtered strain tensor can be directly expressed via a second moment as $\bar{S}_{i j}=\left(2 \rho c_{s}^{2} \tau_{L}^{\star}\right)^{-1} \sum_{a} \mathbf{e}_{\alpha i} \mathbf{e}_{\alpha j}\left(\bar{f}_{\alpha}-\bar{f}_{\alpha}^{e q}\right)$, from which eventually $\tau_{t}=$ $\frac{1}{2}\left[\left(\tau_{L}^{2}+18 \sqrt{2}\left(\rho \Delta x^{2} / \Delta t^{2}\right)^{-1} C_{s}^{2} \Delta x \bar{S}\right)^{1 / 2}-\tau_{L}\right]$ can be derived.

Since the utilization of uniform lattices is not effective for real-world CFD simulations, the LBM has been incorporated into the AMROC software (Deiterding 2011), which supports blockstructured adaptive mesh refinement (SAMR) algorithms generically. In order to fit smoothly into the existing, fully parallelized software, we have implemented the LBM cell-based, which makes the scheme also conservative in $\rho$ and $\rho u_{i}$. In the SAMR approach, finite volume cells are clustered with a special algorithm into non-overlapping rectangular grids. The grids have a suitable layer of halo cells for synchronization and applying inter-level and physical boundary conditions. Refinement levels are integrated recursively. Distributions streaming across refinement boundaries during the recursive temporal update are considered and employed in the collision step of the LBM by a correction-type algorithm that was especially designed for the SAMR methodology, cf. (Deiterding and Wood 2015).

In AMROC, geometrically complex, triangulated boundaries are first transformed into a scalar level set function that stores the distance to the boundary surface. In order to implement nonCartesian boundary conditions with the LBM, we have chosen to adopt for now a generic 1st order accurate ghost fluid approach that was already available in AMROC (Deiterding 2011). Applied to LBM, this technique adjusts the distributions in embedded ghost cells to consider the boundary conditions of a non-Cartesian reflective wall, which involves interpolation and mirroring of $\rho, \mathbf{u}$ across the boundary to $\rho^{\prime}$ and $\overline{\mathbf{u}}$ and modification of the macro-velocity in the immersed boundary 


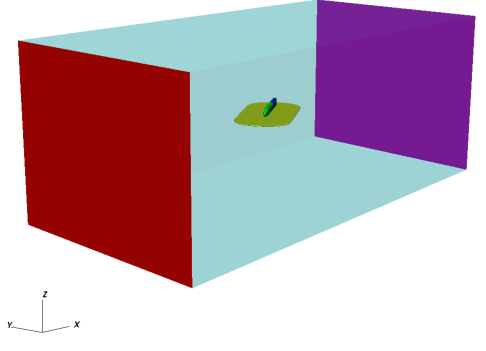

(a)
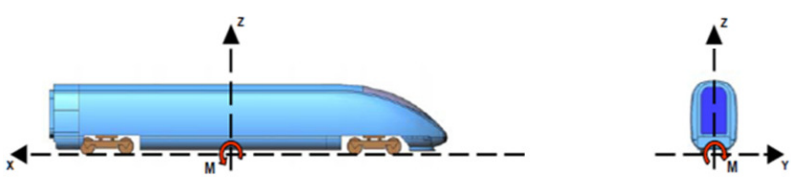

a) Side

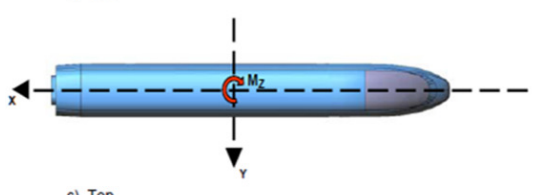

(b)

Figure 1. (a) Global domain. (b) Train coordinate system used for force and moment calculation.

cells to $\mathbf{u}^{\prime}=-\overline{\mathbf{u}}$. From the newly constructed macroscopic values the density distributions in the embedded ghost cells are simply set to $f_{\alpha}^{e q}\left(\rho^{\prime}, \mathbf{u}^{\prime}\right)$ before computing a normal LBM update sweep.

\section{SETUP}

A sketch of the computational domain is given in Fig. 1. For OpenFOAM we apply a freestream velocity normal to the inlet patch (red) of $u_{\infty}=33.1 \mathrm{~m} / \mathrm{s}$ together with zero gradient extrapolation (ZGE) for the pressure. Further, we adopt a constant molecular viscosity for air of $\nu=1.589$. $10^{-5} \mathrm{~m}^{2} / \mathrm{s}$. The Reynolds number is then calculated as $\operatorname{Re}=u_{\infty} H / \nu=250,000$, where $H=12 \mathrm{~cm}$ is used as reference. For the DDES calculations $\nu_{t} / \nu=10^{2}$ is applied. For the LBM computations, we use an inlet velocity of $u_{\infty}=41.93 \mathrm{~m} / \mathrm{s}$ but adjusted viscosity to obtain the same Reynolds number. At domain outlet patches (magenta \& turquoise) we adopt standard outflow boundary conditions in both codes. Zero gradient extrapolation boundary conditions are used on side walls for $\mathrm{OF}$ and non-reflective outflow boundary conditions in the LBM. No-slip boundary conditions are applied at the train and plate surfaces (blue, green, yellow).

As initial data for the OF simulations, we employ results of a RANS computation, which ran for $\sim 10,000$ iterations. For LBM, the initial field of distribution functions is set using the equilibrium function (5), with the velocity set to $(41.93,0,0)^{T} \mathrm{~m} / \mathrm{s}$ and density $\rho_{\infty}=1.13 \mathrm{~kg} / \mathrm{m}^{3}$. Integral force coefficients $c_{f, x / y}=F_{x, y} / q$ are normalized using $q=\frac{1}{2} u_{\infty}^{2} \rho_{\infty} A$, where as reference train cross sectional area $A=0.016 \mathrm{~m}^{2}$ is set ( $\rho_{\infty}=1$ for the incompressible OF calculations). The roll moment coefficient around the train longitudinal axis (cf. Fig. 1, right) is given as $c_{m, x}=M_{x} /(q H)$. Time is expressed in units of convective timescales $T_{C}=H / u_{\infty}$.

Hybrid tetrahedra-prism meshes with $\sim 34 M$ and $\sim 220 M$ cells with ad-hoc refinement around the front car and in the near wake were used for OF. The resolution in the wake is $\Delta x=3 \mathrm{~mm}$ and $\Delta x=1.5 \mathrm{~mm}$, respectively. In addition, 20 Prism layers with a growth factor of 1.2 have been employed around the front car and plate surfaces (cf. Fig. 2, middle). This yields a dimensionless wall normal distance of the first cell of $y^{+}=1.7\left(y^{+}=3.2\right.$ for the small mesh) and normalized wall horizontal cell sizes of $z^{+}=x^{+} \sim 140\left(z^{+}=x^{+} \sim 313\right)$, calculated as $x^{+}=\Delta x \cdot y^{+} / \Delta y$ and $\Delta y$ being the absolute height of the first cell. The OF time steps were $d t / T_{C}=7.5 \cdot 10^{-4}$ (low resolution) and $d t / T_{C}=5.9 \cdot 10^{-4}$ (high resolution). This is associated with a maximal Courant number of $\mathrm{Co}=|\mathbf{u}| \Delta t / \Delta x=50$ (based on the smallest cells).

The SAMR-LBM computation applies a resolution of $\Delta x_{0}=14.97 \mathrm{~mm}$ on the coarsest level and five additional levels refined by a factor of 2 near boundary surfaces. Up to 4 refinement levels are employed in regions of high vorticity based on a criterion evaluated at runtime. A representative depiction of cuts through the resulting mesh is given in the right graphic of Fig. 2. The corresponding normalized wall normal distance of the first cell near the boundary is $y^{+} \sim 43$, which 


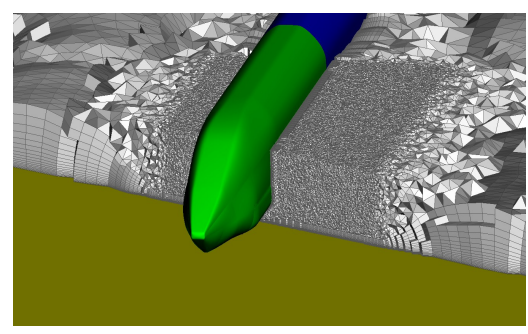

(a)

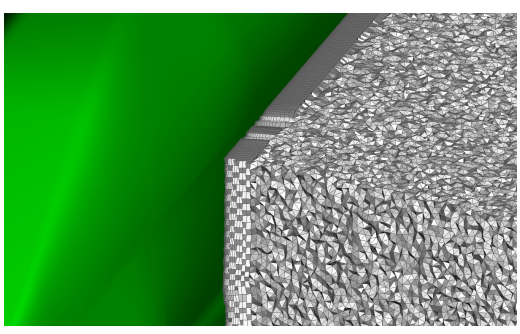

(b)

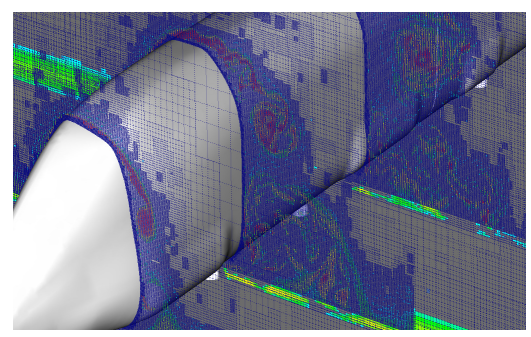

(c)

Figure 2. Mesh used in the OpenFOAM simulations (a,b) versus LBM-SAMR mesh generated by runtime mesh adaptation in $\operatorname{AMROC}(\mathrm{c})$.

\begin{tabular}{lcccc}
\hline & Mesh & $c_{f, x}$ & $c_{f, y}$ & $c_{m, x}$ \\
\hline Wind tunnel & - & -0.06 & -5.28 & -3.46 \\
DDES & low & -0.39 & -5.42 & -3.59 \\
DDES & high & -0.44 & -5.74 & -3.80 \\
LES & high & -0.43 & -6.06 & -4.13 \\
LBM & - & -0.30 & -5.09 & -3.46 \\
\hline
\end{tabular}

Table 1. Integral force and moment coefficients.

\begin{tabular}{lccc}
\hline & viscous & pressure & total \\
\hline$c_{f, x}$ & 0.10 & -0.49 & -0.39 \\
$c_{f, y}$ & -0.04 & -5.38 & -5.42 \\
$c_{m, x}$ & -0.05 & -3.54 & -3.59 \\
\hline
\end{tabular}

Table 2. Viscous and pressure force contributions for DDES (low resolution mesh).

would require wall functions for the correct treatment of the wall boundary layer and calculation of viscous forces. Forces and moments for the LBM are therefore so far computed only for the pressure contribution. The time step applied for LBM on the finest level is $d t / T_{C}=2.7 \cdot 10^{-4}$, and hence the time resolution is about a factor of two higher near geometry surfaces than in the OpenFOAM simulations; however, in regions capturing vortical structures, where a maximum of 4 additional refinement levels are applied, the time resolution is comparable.

\section{RESULTS}

The OF calculations ran on 280 ( 80 for the small mesh) Intel-Xeon cores for $\sim 2500 \mathrm{~h}$ wall clock time $(\sim 622 \mathrm{~h})$, after which they had reached a total physical runtime of $\sim 37-40 T_{C}\left(\sim 57 T_{C}\right)$. On the other hand, the LBM ran on 200 such cores for $\sim 173 \mathrm{~h}$ wall clock time on a final mesh size of $147 \mathrm{M}$ cells, after which it had reached a total physical runtime of $\sim 35 T_{C}$. About $20 \%$ of the overall computational time in the LBM code is spent on dynamic mesh adaption. Hence for a fair comparison we switched off on-the-fly mesh adaption when measuring the performance of AMROC and restarted the computation with the latest SAMR mesh. In this mode, it takes about $0.003 \mathrm{~h}$ CPU to update $1 \mathrm{M}$ cells with their respective time steps. In contrast, OF takes $\sim 0.05 \mathrm{~h} \mathrm{CPU}$ to update $1 \mathrm{M}$ cells of the high resolution mesh and $\sim 0.03 \mathrm{~h} \mathrm{CPU}$ for the low resolution meshes. Hence, the AMROC-LBM implementation is a factor 15-16 faster on comparable meshes.

Integral forces and moments have been averaged over the last 10 convective timescales for the OF simulations and 14 for the LBM. Table 1 displays calculated force and moment coefficients with respect to the coordinate system of Fig. 1, right. Note that the LBM shows only the pressure forces, 

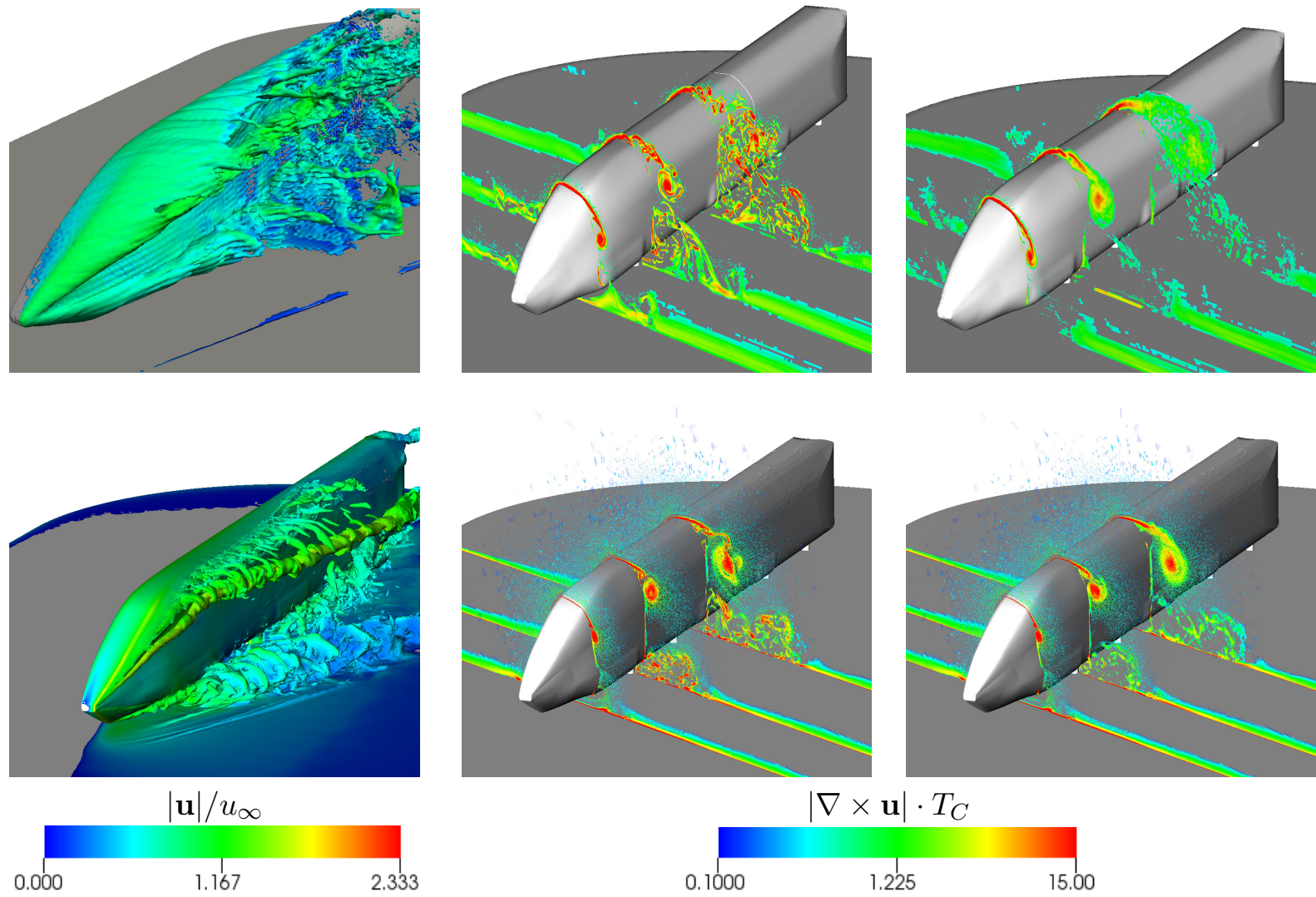

Figure 3. Left: Instantaneous snapshot of the total pressure isosurface $\left(p_{t o t}=0\right)$ with color coding indicating normalized velocity. Middle and right: Normalized vorticity at three different slices, middle: $t=10 T_{C}$, right: time averaged profile. Top row: LBM; lower row: OpenFOAM.

while viscous forces have been neglected. On the other hand, total forces (viscous plus pressure) are displayed for the OF simulations. Table 2 shows their contributions to the overall force and moment coefficients in case of the small DDES simulation. It is evident that the viscous portion is only a small correction to the overturning force and moment coefficient, $c_{f, x}$ and $c_{m, x}$, respectively. Comparing to the wind tunnel data, the LBM delivers very accurate results for $c_{f, y}$ and $c_{m, x}$, with relative discrepancies of $1 \%-3 \%$ (adopting the small viscous correction of Table 2). While the low resolution DDES simulation shows quite accurate predictions as well, the relatively large difference in the high resolution case (i.e. 8\%) may indicate that it is in general difficult to obtain mesh convergence with DDES. The drag force coefficient $c_{f, x}$ is overestimated in all simulations compared to the experiment.

The left column of Fig. 3 shows instantaneous snapshots of the total pressure isosurface for a value of $p_{t o t}=p-p_{\infty}+\frac{1}{2} \rho \mathbf{u}^{2}=0$, while colors indicate the normalized velocity magnitude, i.e. $|\mathbf{u}| / u_{\infty}$. A complex vortex system has formed on the leeward side of the train, where various substructures may be identified. One vortex is triggered at the front nose tip and moves along the roof/leeward interface of the train. It remains steady and attached to the train surface for a length of about two train heights measured from the front nose tip. Vortices passing over the front-nose section of the train seem to spiral in and join this vortex. For OpenFOAM this only seems to occur at the end of the front nose section, while the LBM shows a continues flux into this vortex over the entire front nose.

After detachment this vortex becomes highly unstable, breaking up into small turbulent eddies, as may be seen from the vorticity graphics of Fig. 3. An instantaneous snapshot as well as time averaged data is displayed. Latter has been averaged over $\sim 12$ convective timescales for LBM, 
while averaging over $\sim 5-10 T_{C}$ took place for the high-resolution OF simulation. As may be seen from Fig. 3 (backend slice), the main vortex originating from the front nose is breaking up entirely in the LBM simulations, while the vortex core seems to be conserved in the OF simulation. This may be attributed to the extremely low dissipation of the LBM simulation, thereby allowing small turbulent eddies to be sustained against numerical diffusion. Also, the LBM simulation employs about $60 \%$ higher spatial resolution in the vortex region compared to the OF simulation. From the time averaged data, however, the vortex core can still be identified in the LBM simulation, indicating that a coherent vortex structure manifests itself beyond the turbulent fluctuations.

Besides this major vortex, other smaller vortices, which are also triggered at the front nose of the train, seem to stay attached to the train surface and move all the way along the roof/leeward interface. Unsteady vortex shedding is observed from the train underfloor region, where two distinct sets may be identified in Fig. 3. Interaction with the front bogie cavity causes vortices to be shed in the streamwise direction, while interaction with the back bogie section causes vortices in the train longitudinal direction.

\section{CONCLUSIONS}

For a static nonuniform mesh we have shown that the LBM implementation in AMROC is about a factor of 16 faster for a time step update than OpenFOAM solvers on meshes of similar cell count. This result is not particularly surprising as the LBM is explicit, while the implicit algorithms in OpenFOAM involve solving large linear systems.

We have demonstrated that the LBM delivers very accurate predictions for the overturning force and moment, $c_{f, y}$ and $c_{m, x}$ respectively, with relative discrepancies to the wind tunnel data of $\sim 3 \%$. The high resolution DDES simulation shows somewhat larger discrepancies, which indicates that it may be difficult to obtain mesh convergence with DDES. In terms of the flow field, results between LBM and OF look very similar with only a few differences owing to the higher spatial resolution and lower dissipation of the LBM method.

\section{References}

Baker, C., J. Jones, F. Lopez-Calleja, and J. Munday. 2004. "Measurements of the cross wind forces on trains.." J. Wind Eng. E Industrial Aerodynamics 92: 547-563.

Deiterding, R. 2011. "Block-structured adaptive mesh refinement - theory, implementation and application." ESAIM: Proceedings 34: 97-150.

Deiterding, Ralf, and Stephen L. Wood. 2015. "A dynamically adaptive lattice Boltzmann method for predicting wake phenomena in fully coupled wind engineering problems." In Proc. 6th Int. Conf. Coupled Problems in Science and Engineering, edited by B. Schrefler, E. Onate, and M. Papadrakakis. 489-500.

Fragner, M.M., K.A. Weinman, R. Deiterding, U. Fey, and C. Wagner. 2014. "Numerical and Experimental Studies of Train Geometries Subject to Cross Winds.." In Proc. 2nd Int. Conf. Railway Technology, Civil-Comp Press.

Hähnel, D. 2004. Molekulare Gasdynamik. Springer.

Hemida, H., and S. Krajnovic. 2010. "LES study of the influence of the nose shape and yaw angles on flows structures around trains.." J. Wind Eng. 6 Industrial Aerodynamics 98: 34-46.

Hou, S., J. Sterling, S. Chen, and G. D. Doolen. 1996. "A lattice Boltzmann subgrid model for high Reynolds number flows." In Pattern formation and lattice gas automata, Vol. 6 edited by A. T. Lawniczak and R. Kapral. 151-166. Fields Inst Comm.

Spalart, P., S. Deck, M. Shur, K. Squires, M. Strelets, and A.. Travin. 2006. "A new version of detached-eddy simulation, resistant to ambiguous grid densities.." Theoretical and Computational Fluid Dynamics. 20: $181-195$.

Winter, J. 2013. http://www.dlr.de/dlr/desktopdefault.aspx/tabid-10467/740_read-916/gallery/ 2043. 


\section{List of Tables}

Table 1: Integral force and moment coefficients.

Table 2 : Viscous and pressure force contributions for DDES (low resolution mesh).

\section{List of Figures}

Fig. 1 : (a) Global domain. (b) Train coordinate system used for force and moment calculation.

Fig. 2 : Mesh used in the OpenFOAM simulations (a,b) versus LBM-SAMR mesh generated by runtime mesh adaptation in AMROC (c).

Fig. 3 : Left: Instantaneous snapshot of the total pressure isosurface $\left(p_{\text {tot }}=0\right)$ with color coding

indicating normalized velocity. Middle and right: Normalized vorticity at three different slices, middle: $t=10 T_{C}$, right: time averaged profile. Top row: LBM; lower row: OpenFOAM. middle: $t=10 T_{C}$, ligh: tine averaged profle. Top row: LBM; lower row: OpenFOAM:

8

